segment section with drainage must be considered. Unless the cervix is three-quarters dilated and the head is well through the pelvis, vaginal delivery should not be done; even when carried out in this type of case the cervix will be torn.

\section{Caesarean Section}

From what I have said about Caesarean section it might be thought I was a keen believer in the operation. On the contrary, I believe it has done more harm than good. There are many inexperienced practitioners-and among these I include gynaecologists and surgeons who dabble in obstetrics-who know only one way out of a difficult case of midwifery, and that is section. The classical operation is unfortunately a simple one to perform, it allows the consulting specialist to get back to his work in a hurry, it is usually well paid, and if all goes well the patient and her relations are delighted! But a study of large statistical records will show that the death rate from Caesarean section is much higher than that obtained from vaginal delivery. In other words, the man or woman who advises Caesarean section should have a sound knowledge of the mechanism of labour, especially in regard to the action of the uterus. With this knowledge he will often find that a large dose of morphine combined with a thorough rest will to a large extent lower the incidence of the Caesarean operation.

\section{The Use of Drugs}

One important point I want to raise now is the use of oxytocic drugs. I have seen more harm than good result from the use of pituitary extract, but in the absence of disproportion, and when the obstetrician has got the necessary knowledge, he may give two to three units of pituitary extract every hour until ten or twelve units have been given, or until the patient is in labour. I would like to state that my teaching in the Rotunda was not to give the pituitary extract until after the placenta was delivered, and this is the safest advice. But, bearing this advice in mind, the experienced general practitioner may have exceptional instances in which he may prescribe as advised, but never in large doses. I have seen seven cases of ruptured uterus admitted to hospital following the injection of large doses of pituitary extract given under wrong conditions.

Even the use of quinine must be mentioned: this drug is not without a modicum of danger, and a foetal heart audible before has been found inaudible after 10 grains of quinine. Such cases are few and far between, but the danger must be mentioned.

The foetal mortality in connexion with placenta praevia is so great that a warning must be uttered. When the child is really viable and there are no contraindications Caesarean section should be done if a live child is not to be destroyed.

If monsters are diagnosed (and this can often be done by means of radiology), should they be destroyed? Luckily, most monsters are not capable of living, and each case must be dealt with on its merits. The ventricle in hydrocephalus is often tapped to allow delivery, and the baby is usually born dead. Further details in the management of these conditions will be found in textbooks.

I hope the title of my paper has not been misleading, for I have used it as a vehicle to discuss some knotty problems, but if I have brought home the fact that the foetus should practically never be destroyed my object has been fulfilled.

\section{A BULLOUS ERUPTION DUE TO SULPHANILAMIDE}

BY

F. RAY BETTLEY, M.D.

Dermatologist, Cardiff Royal Infirmary; Medical Officer in Charge of Venereal Diseases Clinic, Cardiff Royal

Infirmary

AND

PHILIP SIMON, B.Sc., M.R.C.S., L.R.C.P. Acting Medical Officer, Pontypridd Venereal Diseases Clinic

The following is a case report of a bullous eruption occurring after the administration of sulphanilamide.

\section{Case Report}

A man aged 49 attended the Pontypridd Venereal Diseases Clinic for the first time on February 8, 1939, with a urethral discharge that began on January 18 , following exposure to infection on the 15th. He had treated himself with ol. santali from January 25 to February 2 without apparent effect. He was found to have anterior urethritis, with a whitish mucopurulent discharge; a smear was examined on one occasion but gonococci were not found. Treatment with sulphanilamide, 1 gramme four times daily, was started on February 8. On the 10th he complained of malaise and nausea, and the dosage was reduced to 1 gramme three times daily. The urethral discharge ceased on February 16 and has not reappeared. When seen on February 22 there was slight oedema of the hands and forearms, with a faint morbilliform rash on their extensor surfaces. Treatment was continued, and two days later the condition was unaltered. The patient then defaulted and was not seen again until March 21, when he gave the following further history. On March 1 he stopped work on account of severe swelling and blueness of the face, forearms, and hands, and photophobia and malaise. He took no more tablets after this. On the 3rd blisters appeared on the face, forearms, and hands. These gradually formed crusts, but on the 15th a fresh attack of swelling and blister formation occurred.

On March 21 he was seen to have a profuse bullous eruption on the face, neck, forearms, hands, and trunk. The bullae were round or oval in shape, with clear serous contents, mostly about $1 \mathrm{~cm}$. in diameter ; some, however, were $2.5 \mathrm{~cm}$. The lower half of the face was more affected than the upper half, the forehead being nearly clear. The rash was severe on the back and sides of the neck, but did not extend into the scalp or below the collar level. On the forearms the dorsal surfaces were more affected. The backs of the hands were in the same state as the forearms, while the palms showed no bullae but desquamated in large flakes. The rash on the trunk was limited to about a dozen papulo-bullous elements scattered over the lumbar and gluteal regions. The bullae tended to rupture spontaneously and reveal eroded or ulcerated surfaces, which healed quickly, leaving pitted scars. There was no suppuration, and by April 6 only scarring remained. The patient refused admission to hospital for investigation. He works as a gardener in the open air with his sleeves rolled up and wearing a cap. The parts on which the rash occurred were exactly those which are exposed to light, with the exception of the few lesions on the trunk.

\section{Comments}

Bullous eruptions due to sulphanilamide are rare, though vesicular rashes have often been seen. A case was reported by Hallam (1939) after the administration of M \& B 693, in which the patient had previously received ultra-violetlight treatment, and the rash was more widespread. Tedder (1939) classifies rashes due to sulphanilamide and its congeners into three groups: (1) Eruptions due to sensitization. These are allergic reactions and can be reproduced by administration of a single small dose of the drug (Tedder, Case 1; Schonberg, 1937). (2) Eruptions due to a toxic effect of the drug. These disappear if the 
dosage of the drug is reduced, smaller doses being tolerated (Tedder, Cases 2 and 3). (3) Eruptions due to a combined effect of suphanilamide and light. In the first two groups the rash is more or less generalized; in the third it occurs mainly or wholly on surfaces exposed to light. Our case falls into the last group, since the rash was almost entirely limited to exposed parts and was quite sharply demarcated at the collar and elbow. The patient had been working in the open, but the weather had been dull and changeable, with little direct sunshine. This is possibly a point of some importance.

It is not definitely known exactly what part is played by light in cases belonging to the third group. Schölzke (1938) recorded a series of cases in which the patients were given uleron and exposed to ultra-violet light. No change in light-sensitivity could be demonstrated. A similar series, also with negative results, was mentioned by Eidinow (1939), who used sulphanilamide instead of uleron. From this it is clear that an increase in sensitivity to light is at any rate not a constant sequel to sulphanilamide medication. On the other hand, Newman and Sharlit (1937) and Brunsting (Tedder, 1939) have been able to produce a spread of an already existing rash to unaffected parts by exposing them to ultra-violet light.

As Tedder points out, this may be due to a non-specific effect of the light, producing a slight degree of irritation which determines the site of the rash. This phenomenon is well known to occur in rashes of widely different types, in which irritation due to light seems to produce a locus minoris resistentiae. This might have happened in the case mentioned by Hallam, but in our case exposure to light was so trivial-and this on parts habitually exposed - that even the slightest degree of irritation cannot be presumed. We are therefore bound to assume that lightsensitivity was present. This may have arisen in two ways, as suggested by Newman and Sharlit (1939): either there is a direct light-sensitization caused by the drug, as is produced by fluorescent compounds such as eosin, or sensitization is indirectly due to the drug by the production of porphyrins. The first hypothesis is made unlikely by the experiments of Schölzke and of Eidinow, since if sulphanilamide had this effect it should be regularly observed. Light-sensitization by porphyrins remains and appears to fit the facts; the production of porphyrins is known to occur when sulphanilamide is given, and their quantity to vary considerably in different cases. Unfortunately in neither Hallam's nor our case were examinations made for these substances. Brunsting, however, observed two cases of this type of eruption in which urinary porphyrins were much above normal. It may be remarked that the presence of cyanosis due to porphyrinaemia does not always go hand in hand with light-sensitivity. This is probably due to the fact that different porphyrins have different sensitizing powers when present in the blood stream. The present evidence points, therefore, to porphyrinaemia as the cause of light-sensitization following sulphanilamide medication.

It is of further interest to note that in hydroa aestivale a bullous eruption following exposure to light is associated with haematoporphyrinaemia.

We are indebted to Dr. E. Colston Williams, medical officer of health for Glamorgan, for permission to publish this case.

\section{REFERENCES}

Eidinow, A. (1939). British Medical Journal, 1, 692.

Hallam, R. (1939). Ibid., 1, 559 .

Newman, B. A., and Sharlit, H. (1937). J. Amer. med. Ass., 109, 1036 .

Schölzke, K 1939), Arch. Derm. Syph., Chicago,

Schonberg, I. L. (1937). J. Amer. med. Ass., 109, 1035

Schonberg, I. L. (1937). J. Amer. Med. Ass. 109, 1035.

\section{OBSERVATIONS ON THE AGGLUTINATION TEST FOR WEIL'S DISEASE}

BY

\author{
H. C. BROWN, C.I.E., M.B., D.T.M. \& H. \\ AND
}

J. C. BROOM, M.D.

(From the Wellcome Bureau of Scientific Research, London)

Although in principle this test is essentially similar to a bacterial agglutination test, difficulties have been met with which would mask or invalidate the results in the hands of an inexperienced observer. In the first place, though all the strains of the organism which we have used have been isolated from patients in England whose sera have reacted strongly to the standard London human strain of Leptospira icterohaemorrhagiae, only certain of these strains are suitable for the agglutination test. To take a typical example, five human strains of $L$. icterohaemorrhagiae were subcultivated in Fletcher's broth until the growth in each of the cultures was sufficiently vigorous to be apparently suitable for the agglutination test. The cultures were then formolized to exactly 0.2 per cent. One serum was put up against these five cultures, using Schüffner's technique, in dilutions ranging from 1 in 10 to 1 in 30,000 . The result of this was as follows: one strain showed no agglutination; one was positive up to a serum dilution of 1 in 100 ; one was positive up to 1 in 300 ; and two were positive up to 1 in 1,000 . The technique employed in this case was twenty-four hours' interaction at room temperature. The results were read with a microscope, $2 / 3$-inch objective and $\times 10$ ocular with dark-ground illumination, no cover-slip being used. This serum consistently gave a titre of 1 in 3,000 with our standard formolized culture. By "standard" culture we do not mean that it has been standardized after the manner in which the Oxford bacterial cultures are standardized, but merely that for the last five years it has been known to give consistent and repeatable results.

We have some reason to believe that cultures which have been quite recently formolized are not agglutinated to so high a titre as those formolized for forty-eight hours or more. The question of formolization of the cultures has not presented any difficulty, and we have not met with the acid agglutination referred to by Professor Schüffner. We have always used analytical reagent formalin, and have not added more than 0.2 per cent.

From time to time a macroscopic agglutination technique has been advocated, and there is no doubt that when a suitable antigen can be obtained a florid serum will give a positive result after one hour's incubation. Pot (1936) advocates a macroscopic test, and claims that out of twenty-six known positive sera twenty-five gave a positive reaction with his method. In our experience the macroscopic method is not nearly so sensitive as the microscopical, and we are of opinion that certain sera of low titre, such as those met with shortly after the sixth day of disease, may be missed if the macroscopic test is employed. Using the microscopical procedure the titres obtained by Brown (1935) approximated very closely to those obtained by Professor Schüffner when a series of sera were examined by both observers.

\section{Technique of the Test}

When examining a serum it is necessary to put up a known positive serum in all dilutions at the same time. This will indicate any change in agglutinability of the 